\title{
A Sheaf Model of the Algebraic Closure
}

\author{
Bassel Mannaa Thierry Coquand \\ Department of Computer Science and Engineering \\ University of Gothenburg* \\ Gothenburg, Sweden \\ bassel.mannaa@cse.gu.se thierry.coquand@cse.gu.se
}

\begin{abstract}
In constructive algebra one cannot in general decide the irreducibility of a polynomial over a field $K$. This poses some problems to showing the existence of the algebraic closure of $K$. We give a possible constructive interpretation of the existence of the algebraic closure of a field in characteristic 0 by building, in a constructive metatheory, a suitable site model where there is such an algebraic closure. One can then extract computational content from this model. We give examples of computation based on this model.
\end{abstract}

\section{Introduction}

Since in general it is not decidable whether a given polynomial over a field is irreducible, even when the field is given explicitly [6], the notion of algebraic field extension and consequently the notion of algebraic closure becomes problematic from a constructive point of view. Even in situations where one can constructively assert the existence of an algebraic closure of a field [14, Ch. 6] the computational content of such assertions are not always clear. We present a constructive interpretation of the algebraic closure of field $K$ in characteristic 0 as a site model. Our approach is different from [15] in that we do not assume a polynomial over a field to be decomposable into irreducible factors. The model presented here has a direct computational content and can be viewed as a model of dynamical evaluation in the sense of Duval [5] (see also [4]). The site, described in section 3, is given by the category of finitely presented (von Neumann) regular algebras over $K$ with the appropriate Grothendieck topology. In section 4 we prove that the topos $\mathscr{E}$ of sheaves on this site contains a model of an algebraically closed field extension of $K$. An alternative approach using profinite Galois group is presented in [8]. We also investigate some of the properties of the topos $\mathscr{E}$. Theorem 6.3 shows that the axiom of choice fails to hold in $\mathscr{E}$ whenever $K$ is not algebraically closed. Theorem 6.4 shows that when the base field $K$ is the rationals the weaker axiom of dependent choice fails to hold. We restrict ourselves to constructive metatheory throughout the paper with the exception of section 8 in which we show that in a classical metatheory the topos $\mathscr{E}$ is boolean (Theorem 8.6. As we will demonstrate by Theorem 8.8 this cannot be shown to hold in an intuitionistic metatheory.

\section{Coverage, sheaves, and Kripke-Joyal semantics}

In this section we recall some notions that we will use in the remainder the paper, mostly following the presentation in [7]. A coverage on a category $\mathscr{C}$ is a function $\mathbf{J}$ assigning to each object $C$ of $\mathscr{C}$ a collection $\mathbf{J}(C)$ of families of morphisms with codomain $C$ such that for any $\left\{f_{i}: C_{i} \rightarrow C\right\}_{i \in I} \in \mathbf{J}(C)$ and morphism $g: D \rightarrow C$ of $\mathscr{C}$ there exist $\left\{h_{j}: D_{j} \rightarrow D\right\}_{j \in J} \in \mathbf{J}(D)$ such that for each $j \in J$ the morphism

${ }^{*}$ The research leading to this work has been supported by ERC Advanced grant project 247219.

Paulo Oliva (Ed.): Classical Logic and Computation 2014

EPTCS 164, 2014, pp. 18-32 doi $10.4204 /$ EPTCS.164.2 (c) B. Mannaa \& T. Coquand

This work is licensed under the Creative Commons Attribution License. 
$g h_{j}$ factors through $f_{\ell}$ for some $\ell \in I$. A family $S \in \mathbf{J}(C)$ is called elementary cover or elementary covering family of $C$. A site is a category with coverage $(\mathscr{C}, \mathbf{J})$. For a presheaf $\mathbf{P}: \mathscr{C}^{o p} \rightarrow$ Set and family $S=\left\{g_{i}: A_{i} \rightarrow A\right\}_{i \in I}$ of morphisms of $\mathscr{C}$ we say that a family $\left\{s_{i} \in \mathbf{P}\left(A_{i}\right)\right\}_{i \in I}$ is compatible if for each $\ell, j \in I$ whenever we have $h: B \rightarrow A_{\ell}$ and $f: B \rightarrow A_{j}$ such that $g_{\ell} h=g_{j} f$ then $s_{\ell} h=s_{j} f$, where by $s_{\ell} h$ we mean the restriction of $s_{\ell}$ along $h$, i.e. $\mathbf{P}(h) s_{\ell}$. A presheaf $\mathbf{P}$ is a sheaf on the site $(\mathscr{C}, \mathbf{J})$ if for any object $C$ and any $\left\{f_{i}: C_{i} \rightarrow C\right\}_{i \in I} \in \mathbf{J}(C)$ if $\left\{s_{i} \in \mathbf{P}\left(C_{i}\right)\right\}_{i \in I}$ is compatible then there exist a unique $s \in \mathbf{P}(C)$ such that $s f_{i}=s_{i}$. We call such $s$ the amalgamation of $\left\{s_{i}\right\}_{i \in I}$. Let $\mathbf{J}$ be a coverage on $\mathscr{C}$ we define a closure $\mathbf{J}^{*}$ of $\mathbf{J}$ as follows: For all objects $C$ of $\mathscr{C}$ i. $\left\{C \stackrel{{ }^{1} C}{\longrightarrow} C\right\} \in \mathbf{J}^{*}(C)$, ii. If $S \in \mathbf{J}(C)$ then $S \in \mathbf{J}^{*}(C)$, and, iii. If $\left\{C_{i} \stackrel{f_{i}}{\longrightarrow} C\right\}_{i \in I} \in \mathbf{J}^{*}(C)$ and for each $i \in I,\left\{C_{i j} \stackrel{g_{i j}}{\longrightarrow} C_{i}\right\}_{j \in J_{i}} \in \mathbf{J}^{*}\left(C_{i}\right)$ then $\left\{C_{i j} \stackrel{f_{i} g_{i j}}{\longrightarrow} C\right\}_{i \in I, j \in J_{i}} \in \mathbf{J}^{*}(C)$. A family $T \in \mathbf{J}^{*}(C)$ is called cover or covering family of $C$.

We work with a typed language with equality $\mathscr{L}\left[V_{1}, \ldots, V_{n}\right]$ having the basic types $V_{1}, \ldots, V_{n}$ and type formers $-\times-,(-)^{-}, \mathscr{P}(-)$. The language $\mathscr{L}\left[V_{1}, \ldots, V_{n}\right]$ has typed constants and function symbols. For any type $Y$ one has a stock of variables $y_{1}, y_{2}, \ldots$ of type $Y$. Terms and formulas of the language are defined as usual. We work within the proof theory of intuitionistic higher-order logic (IHOL). A detailed description of this deduction system is given in [1].

The language $\mathscr{L}\left[V_{1}, \ldots, V_{n}\right]$ along with deduction system IHOL can be interpreted in an elementary topos in what is referred to as topos semantics. For a sheaf topos this interpretation takes a simpler form reminiscent of Beth semantics, usually referred to as Kripke-Joyal sheaf semantics. We describe this semantics here briefly following [15].

Let $\mathscr{E}=\operatorname{Sh}(\mathscr{C}, \mathbf{J})$ be a sheaf topos. An interpretation of the language $\mathscr{L}\left[V_{1}, \ldots, V_{n}\right]$ in the topos $\mathscr{E}$ is given as follows: Associate to each basic type $V_{i}$ of $\mathscr{L}\left[V_{1}, \ldots, V_{n}\right]$ an object $\mathbf{V}_{i}$ of $\mathscr{E}$. If $Y$ and $Z$ are types of $\mathscr{L}\left[V_{1}, \ldots, V_{n}\right]$ interpreted by objects $\mathbf{Y}$ and $\mathbf{Z}$, respectively, then the types $Y \times Z, Y^{Z}, \mathscr{P}(Z)$ are interpreted by $\mathbf{Y} \times \mathbf{Z}, \mathbf{Y}^{\mathbf{Z}}, \Omega^{\mathbf{Z}}$, respectively, where $\Omega$ is the subobject classifier of $\mathscr{E}$. A constant $e$ of type $E$ is interpreted by an arrow $\mathbf{1} \stackrel{\mathbf{e}}{\rightarrow} \mathbf{E}$ where $\mathbf{E}$ is the interpretation of $E$. For a term $\tau$ and an object $\mathbf{X}$ of $\mathscr{E}$, we write $\tau: \mathbf{X}$ to mean $\tau$ has a type $X$ interpreted by the object $\mathbf{X}$.

Let $\phi\left(x_{1}, \ldots, x_{n}\right)$ be a formula with variables $x_{1}: \mathbf{X}_{1}, \ldots, x_{n}: \mathbf{X}_{n}$. Let $c_{1} \in \mathbf{X}_{j}(C), \ldots, c_{n} \in \mathbf{X}_{n}(C)$ for some object $C$ of $\mathscr{C}$. We define the relation $C$ forces $\phi\left(x_{1}, \ldots, x_{n}\right)\left[c_{1}, \ldots, c_{n}\right]$ written $C \Vdash \phi\left(x_{1}, \ldots, x_{n}\right)\left[c_{1}, \ldots, c_{n}\right]$ by induction on the structure of $\phi$.

Definition 2.1 (Forcing). First we replace the constants in $\phi$ by variables of the same type as follows: Let $e_{1}: \mathbf{E}_{1}, \ldots, e_{m}: \mathbf{E}_{m}$ be the constants in $\phi\left(x_{1}, \ldots, x_{n}\right)$ then $C \Vdash \phi\left(x_{1}, \ldots, x_{n}\right)\left[c_{1}, \ldots, c_{n}\right]$ iff

$$
C \Vdash \phi\left[y_{1} / e_{1}, \ldots, y_{m} / e_{m}\right]\left(y_{1}, \ldots, y_{m}, x_{1}, \ldots, x_{n}\right)\left[\mathbf{e}_{1_{C}}(*), \ldots, \mathbf{e}_{m_{C}}(*), c_{1}, \ldots, c_{n}\right]
$$

where $y_{i}: \mathbf{E}_{i}$ and $\mathbf{e}_{i}: \mathbf{1} \rightarrow \mathbf{E}_{i}$ is the interpretation of $e_{i}$.

Now it suffices to define the forcing relation for formulas free of constants by induction as follows:

$\mathrm{T} C \Vdash \top$.

$\perp C \Vdash \perp$ iff the empty family is a cover of $C$.

$C \Vdash\left(x_{1}=x_{2}\right)\left[c_{1}, c_{2}\right]$ iff $c_{1}=c_{2}$.

$\wedge C \Vdash(\phi \wedge \psi)\left(x_{1}, \ldots, x_{n}\right)\left[c_{1}, \ldots, c_{n}\right]$ iff $C \Vdash \phi\left(x_{1}, \ldots, x_{n}\right)\left[c_{1}, \ldots, c_{n}\right]$ and $C \Vdash \psi\left(x_{1}, \ldots, x_{n}\right)\left[c_{1}, \ldots, c_{n}\right]$.

$\mathrm{V} C \Vdash(\phi \vee \psi)\left(x_{1}, \ldots, x_{n}\right)\left[c_{1}, \ldots, c_{n}\right]$ iff there exist a cover $\left\{C_{i} \stackrel{f_{i}}{\longrightarrow} C\right\}_{i \in I} \in \mathbf{J}^{*}(C)$ such that $C_{i} \Vdash \phi\left(x_{1}, \ldots, x_{n}\right)\left[c_{1} f_{i}, \ldots, c_{n} f_{i}\right]$ or $C_{i} \Vdash \psi\left(x_{1}, \ldots, x_{n}\right)\left[c_{1} f_{i}, \ldots, c_{n} f_{i}\right]$ for each $i \in I$.

$\Rightarrow C \Vdash(\phi \Rightarrow \psi)\left(x_{1}, \ldots, x_{n}\right)\left[c_{1}, \ldots, c_{n}\right]$ iff for every morphism $f: D \rightarrow C$ whenever $D \Vdash \phi\left(x_{1}, \ldots, x_{n}\right)\left[c_{1} f, \ldots, c_{n} f\right]$ one has $D \Vdash \psi\left(x_{1}, \ldots, x_{n}\right)\left[c_{1} f, \ldots, c_{n} f\right]$. 
Let $y$ be a variable of the type $Y$ interpreted by the object $\mathbf{Y}$ of $\mathscr{E}$.

$\exists C \Vdash\left(\exists y \phi\left(x_{1}, \ldots, x_{n}, y\right)\right)\left[c_{1}, \ldots, c_{n}\right]$ iff there exist a cover $\left\{C_{i} \stackrel{f_{i}}{\longrightarrow} C\right\}_{i \in I} \in \mathbf{J}^{*}(C)$ such that for each $i \in I$ one has $C_{i} \Vdash \phi\left(x_{1}, \ldots, x_{n}, y\right)\left[c_{1} f_{i}, \ldots, c_{n} f_{i}, d\right]$ for some $d \in \mathbf{Y}\left(C_{i}\right)$.

$\forall C \Vdash\left(\forall y \phi\left(x_{1}, \ldots, x_{n}, y\right)\right)\left[c_{1}, \ldots, c_{n}\right]$ iff for every morphism $f: D \rightarrow C$ and for all $d \in \mathbf{Y}(D)$ one has $D \Vdash \phi\left(x_{1}, \ldots, x_{n}, y\right)\left[c_{1} f, \ldots, c_{n} f, d\right]$.

We have the following derivable local character and monotonicity laws:

LC If $\left\{C_{i} \stackrel{f_{i}}{\rightarrow} C\right\}_{i \in I} \in \mathbf{J}^{*}(C)$ and for all $i \in I, C_{i} \Vdash \phi\left(x_{1}, \ldots, x_{n}\right)\left[c_{1} f_{i}, \ldots, c_{n} f_{i}\right]$ then $C \Vdash \phi\left(x_{1}, \ldots, x_{n}\right)\left[c_{1}, \ldots, c_{n}\right]$.

$\mathrm{M}$ If $C \Vdash \phi\left(x_{1}, \ldots, x_{n}\right)\left[c_{1}, \ldots, c_{n}\right]$ and $f: D \rightarrow C$ then $D \Vdash \phi\left(x_{1}, \ldots, x_{n}\right)\left[c_{1} f, \ldots, c_{n} f\right]$.

\section{The topos $\operatorname{Sh}\left(\mathscr{R} \mathscr{A}_{K}^{o p}, \mathbf{J}\right)$}

Definition 3.1 (Regular ring). A commutative ring $R$ is (von Neumann) regular if for every element $a \in R$ there exist $b \in R$ such that $a b a=a$ and $b a b=b$. This element $b$ is called the quasi-inverse of $a$.

The quasi-inverse of an element $a$ is unique for $a[9, \mathrm{Ch} .4]$. We thus use the notation $a^{*}$ to refer to the quasi-inverse of $a$. A ring is regular iff it is zero-dimensional and reduced. To be regular is equivalent to the fact that any principal ideal (consequently, any finitely generated ideal) is generated by an idempotent. If $R$ is regular and $a \in R$ then $e=a a^{*}$ is an idempotent such that $\langle e\rangle=\langle a\rangle$ and $R$ is isomorphic to $R_{0} \times R_{1}$ with $R_{0}=R /\langle e\rangle$ and $R_{1}=R /\langle 1-e\rangle$. Furthermore $a$ is 0 on the component $R_{0}$ and invertible on the component $R_{1}$.

Definition 3.2 (Fundamental system of orthogonal idempotents). A family $\left(e_{i}\right)_{i \in I}$ of idempotents in a ring $R$ is a fundamental system of orthogonal idempotents if $\sum_{i \in I} e_{i}=1$ and $\forall i, j\left[i \neq j \Rightarrow e_{i} e_{j}=0\right]$.

Lemma 3.3. Given a fundamental system of orthogonal idempotents $\left(e_{i}\right)_{i \in I}$ in a ring $A$ we have a decomposition $A \cong \prod_{i \in I} A /\left\langle 1-e_{i}\right\rangle$.

Proof. Follows by induction from the fact that $A \cong A /\langle e\rangle \times A /\langle 1-e\rangle$ for an idempotent $e \in A$.

Definition 3.4 (Separable polynomial). Let $R$ be a ring. A polynomial $p \in R[X]$ is separable if there exist $r, s \in R[X]$ such that $r p+s p^{\prime}=1$, where $p^{\prime} \in R[X]$ is the derivative of $p$.

Definition 3.5. A ring $R$ is a (strict) Bézout ring if for all $a, b \in R$ we can find $g, a_{1}, b_{1}, c, d \in R$ such that $a=a_{1} g, b=b_{1} g$ and $c a_{1}+d b_{1}=1$ [9, Ch. 4].

If $R$ is a regular ring then $R[X]$ is a strict Bézout ring (and the converse is true [9]). Intuitively we can compute the gcd as if $R$ was a field, but we may need to split $R$ when deciding if an element is invertible or 0 . Using this, we see that given $a, b$ in $R[X]$ we can find a decomposition $R_{1}, \ldots, R_{n}$ of $R$ and for each $i$ we have $g, a_{1}, b_{1}, c, d$ in $R_{i}[X]$ such that $a=a_{1} g, b=b_{1} g$ and $c a_{1}+d b_{1}=1$ with $g$ monic.

Lemma 3.6. If $R$ is regular and $p$ in $R[X]$ is a separable polynomial then $R[a]=R[X] /\langle p\rangle$ is regular.

Proof. If $c=q(a)$ is an element of $R[a]$ with $q$ in $R[X]$ we compute the $\operatorname{gcd} g$ of $p$ and $q$. If $p=g p_{1}$, we can find $u$ and $v$ in $R[X]$ such that $u g+v p_{1}=1$ since $p$ is separable. We then have $g(a) p_{1}(a)=0$ and $u(a) g(a)+v(a) p_{1}(a)=1$. It follows that $e=u(a) g(a)$ is idempotent and we have $\langle e\rangle=\langle g(a)\rangle$. 
An algebra $A$ over a field $K$ is finitely presented if it is of the form $K\left[X_{1}, . ., X_{n}\right] /\left\langle f_{1}, \ldots, f_{m}\right\rangle$, i.e. the quotient of the polynomial ring over $K$ in finitely many variables by a finitely generated ideal.

In order to build the classifying topos of a coherent theory $T$ it is customary in the literature to consider the category of all finitely presented $T_{0}$ algebras where $T_{0}$ is an equational subtheory of $T$. The axioms of $T$ then give rise to a coverage on the dual category [11, Ch. 9]. For our purpose consider the category $\mathscr{C}$ of finitely presented $K$-algebras. Given an object $R$ of $\mathscr{C}$, the axiom schema of algebraic closure and the field axiom give rise to families (i.) $R \rightarrow R[X] /\langle p\rangle$ where $p \in R[X]$ is monic and

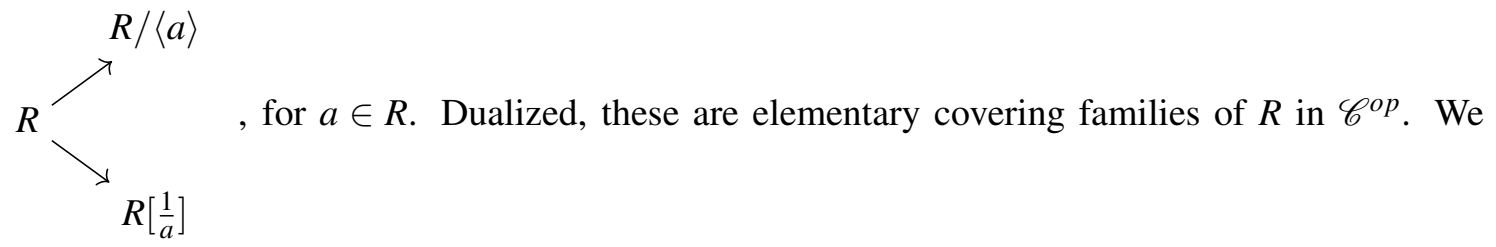

observe however that we can limit our consideration only to those finitely presented $K$-algebras that are zero dimensional and reduced, i.e. regular. In this case we can assume $a$ is an idempotent and we only consider extensions $R[X] /\langle p\rangle$ where $p$ is separable.

Let $\mathscr{R} \mathscr{A}_{K}$ be the small category of finitely presented regular algebras over a fixed field $K$ and $K$ homomorphisms. First we fix an countable set of names $S$. An object of $\mathscr{R} \mathscr{A}_{K}$ is a regular algebra of the form $K\left[X_{1}, \ldots, X_{n}\right] /\left\langle f_{1}, \ldots, f_{m}\right\rangle$ where $X_{i} \in S$ for all $1 \leq i \leq n$. Note that for any object $R$, there is a unique morphism $K \rightarrow R$. A finitely presented regular $K$-algebra $A$ is a finite dimensional $K$-algebra, i.e. $A$ has a finite dimension as a vector space over $K$ [9, Ch 4, Theorem 8.16]. The trivial ring 0 is the terminal object in the category $\mathscr{R} \mathscr{A}_{K}$ and $K$ is its initial object.

To specify a coverage $\mathbf{J}$ on the category $\mathscr{R} \mathscr{A}_{K}^{o p}$, we define for each object $A$ a collection $\mathbf{J}^{o p}(A)$ of families of morphisms of $\mathscr{R} \mathscr{A}_{K}$ with domain $A$. We then take $\mathbf{J}(A)$ to be the dual of $\mathbf{J}^{o p}(A)$ in the sense that $\left\{\overline{\varphi_{i}}: A_{i} \rightarrow A\right\}_{i \in I} \in \mathbf{J}(A)$ if and only if $\left\{\varphi_{i}: A \rightarrow A_{i}\right\}_{i \in I} \in \mathbf{J}^{o p}(A)$ where $\varphi_{i}$ of $\mathscr{R} \mathscr{A}_{K}$ is the dual of $\bar{\varphi}_{i}$ of $\mathscr{R} \mathscr{A}_{K}^{o p}$. We call $\mathbf{J}^{o p}$ cocoverage. We call an element of $\mathbf{J}^{o p}(A)$ an elementary cocover (cocovering family) of $A$. We define $\mathbf{J}^{* o p}$ similarly. We call elements of $\mathbf{J}^{* o p}(A)$ cocovers (cocovering families) of $A$. By a separable extension of a ring $R$ we mean a ring $R[a]=R[X] /\langle p\rangle$ where $p \in R[X]$ is non-constant, monic and separable.

Definition 3.7 (Topology for $\mathscr{R} \mathscr{A}_{K}^{o p}$ ). For an object $A$ of $\mathscr{R} \mathscr{A}_{K}$ the cocovering families are given by:

(i.) If $\left(e_{i}\right)_{i \in I}$ is a fundamental system of orthogonal idempotents of $A$, then $\left\{A \stackrel{\varphi_{i}}{\longrightarrow} A /\left\langle 1-e_{i}\right\rangle\right\}_{i \in I} \in$ $\mathbf{J}^{o p}(A)$ where for each $i \in I, \varphi_{i}$ is the canonical homomorphism.

(ii.) Let $A[a]$ be a separable extension of $A$. We have $\{A \stackrel{\vartheta}{\rightarrow} A[a]\} \in \mathbf{J}^{o p}(A)$ where $\vartheta$ is the canonical embedding.

Note that in particular 3.7|(i.) implies that the trivial algebra 0 is covered by the empty family of morphisms since an empty family of elements in this ring form a fundamental system of orthogonal idempotents. Also note that 3.7 (ii.) implies that $\left\{A \stackrel{1_{A}}{\longrightarrow} A\right\} \in \mathbf{J}^{o p}(A)$.

Lemma 3.8. The function $\mathbf{J}$ of Definition 3.7 is a coverage on $\mathscr{R} \mathscr{A}_{K}^{o p}$.

Proof. Let $\eta: R \rightarrow A$ be a morphism of $\mathscr{R} \mathscr{A}_{K}$ and $S \in \mathbf{J}^{o p}(R)$. We show that there exist an elementary cocover $T \in \mathbf{J}^{o p}(A)$ such that for each $\vartheta \in T, \vartheta \eta$ factors through some $\varphi \in S$. By duality, this implies $\mathbf{J}$ is a coverage on $\mathscr{R} \mathscr{A}_{K}^{o p}$. By case analysis on the clauses of Definition 3.7 . 
(i.) If $S=\left\{\varphi_{i}: R \rightarrow R /\left\langle 1-e_{i}\right\rangle\right\}_{i \in I}$, where $\left(e_{i}\right)_{i \in I}$ is a fundamental system of orthogonal idempotents of $R$. In $A$, the family $\left(\eta\left(e_{i}\right)\right)_{i \in I}$ is fundamental system of orthogonal idempotents. We have an elementary cocover $\left\{\vartheta_{i}: A \rightarrow A /\left\langle 1-\eta\left(e_{i}\right)\right\rangle\right\}_{i \in I} \in \mathbf{J}^{o p}(A)$. For each $i \in I$, the homomorphism $\eta$ induces a $K$-homomorphism $\eta_{e_{i}}: R /\left\langle 1-e_{i}\right\rangle \rightarrow A /\left\langle 1-\eta\left(e_{i}\right)\right\rangle$ where $\eta_{e_{i}}\left(r+\left\langle 1-e_{i}\right\rangle\right)=\eta(r)+$ $\left\langle 1-\eta\left(e_{i}\right)\right\rangle$. Since $\vartheta_{i}(\eta(r))=\eta(r)+\left\langle 1-\eta\left(e_{i}\right)\right\rangle$ we have that $\vartheta_{i} \eta=\eta_{e_{i}} \varphi_{i}$.

(ii.) If $S=\{\varphi: R \rightarrow R[r]\}$ with $R[r]=R[X] /\langle p\rangle$ and $p \in R[X]$ monic, non-constant, and separable. Since $s p+t p^{\prime}=1$, we have $\eta(s) \eta(p)+\eta(t) \eta\left(p^{\prime}\right)=\eta(s) \eta(p)+\eta(t) \eta(p)^{\prime}=1$. Then $q=\eta(p) \in$ $A[X]$ is separable. Let $A[a]=A[X] /\langle q\rangle$. We have an elementary cocover $\{\vartheta: A \rightarrow A[a]\} \in \mathbf{J}^{o p}(A)$ where $\vartheta$ is the canonical embedding. Let $\zeta: R[r] \rightarrow A[a]$ be the $K$-homomorphism such that $\left.\zeta\right|_{R}=\eta$ and $\zeta(r)=a$. For $b \in R$, we have $\vartheta(\eta(b))=\zeta(\varphi(b))$.

Lemma 3.9. Let $\mathbf{P}: \mathscr{R} \mathscr{A}_{K} \rightarrow$ Set be a presheaf on $\mathscr{R} \mathscr{A}_{K}^{o p}$ such that $\mathbf{P}(0)=1$. Let $R$ be an object of $\mathscr{R} \mathscr{A}_{K}$ and let $\left(e_{i}\right)_{i \in I}$ be a fundamental system of orthogonal idempotents of $R$. For each $i \in I$, let $R_{i}=R /\left\langle 1-e_{i}\right\rangle$ and let $\varphi_{i}: R \rightarrow R_{i}$ be the canonical homomorphism. Any family $\left\{s_{i} \in \mathbf{P}\left(R_{i}\right)\right\}$ is compatible.

Proof. Let $B$ be an object and for some $i, j \in I$ let $\vartheta: R_{i} \rightarrow B$ and $\zeta: R_{j} \rightarrow B$ be such that $\vartheta \varphi_{i}=\zeta \varphi_{j}$. We will show that $\mathbf{P}(\vartheta)\left(s_{i}\right)=\mathbf{P}(\zeta)\left(s_{j}\right)$.

(i.) If $i=j$, then since $\varphi_{i}$ is surjective we have $\vartheta=\zeta$ and $\mathbf{P}(\vartheta)=\mathbf{P}(\zeta)$.

(ii.) If $i \neq j$, then since $e_{i} e_{j}=0, \varphi_{i}\left(e_{i}\right)=1$ and $\varphi_{j}\left(e_{j}\right)=1$ we have $\varphi_{j}\left(e_{i}\right)=\varphi_{j}\left(e_{i} e_{j}\right)=0$. But then

$$
1=\vartheta(1)=\vartheta\left(\varphi_{i}\left(e_{i}\right)\right)=\zeta\left(\varphi_{j}\left(e_{i}\right)\right)=\zeta(0)=0
$$

Hence $B$ is the trivial algebra 0 . By assumption $\mathbf{P}(0)=1$, hence $\mathbf{P}(\vartheta)\left(s_{i}\right)=\mathbf{P}(\zeta)\left(s_{j}\right)=*$.

Corollary 3.10. Let $\mathbf{F}$ be a sheaf on $\left(\mathscr{R} \mathscr{A}_{K}^{o p}, \mathbf{J}\right)$. Let $R$ be an object of $\mathscr{R} \mathscr{A}_{K}$ and $\left(e_{i}\right)_{i \in I}$ a fundamental system of orthogonal idempotents of $R$. Let $R_{i}=R /\left\langle 1-e_{i}\right\rangle$ and $\varphi_{i}: R \rightarrow R_{i}$ be the canonical homomorphism. The map $f: \mathbf{F}(R) \rightarrow \prod_{i \in I} \mathbf{F}\left(R_{i}\right)$ such that $f(s)=\left(\mathbf{F}\left(\varphi_{i}\right) s\right)_{i \in I}$ is an isomorphism.

Proof. Since $\mathbf{F}(0)=1$, by Lemma 3.9 any family $\left\{s_{i} \in \mathbf{F}\left(R_{i}\right)\right\}_{i \in I}$ is compatible. Since $\mathbf{F}$ is a sheaf, the family $\left\{s_{i} \in \mathbf{F}\left(R_{i}\right)\right\}_{i \in I}$ has a unique amalgamation $s \in \mathbf{F}(R)$ with restrictions $s \varphi_{i}=s_{i}$. The isomorphism is given by $f s=\left(s \varphi_{i}\right)_{i \in I}$. We can then use the tuple notation $\left(s_{i}\right)_{i \in I}$ to denote the element $s$ in $\mathbf{F}(R)$.

One say that a polynomial $f \in R[X]$ has a formal degree $n$ if $f$ can be written as $f=a_{n} X^{n}+\ldots+a_{0}$ which is to express that for any $m>n$ the coefficient of $X^{m}$ is known to be 0 .

Lemma 3.11. Let $R$ be a regular ring and $p_{1}, p_{2} \in R[X]$ be monic polynomials of degrees $n_{1}$ and $n_{2}$ respectively. Let $R[a, b]=R[X, Y] /\left\langle p_{1}(X), p_{2}(Y)\right\rangle$. Let $q_{1}, q_{2} \in R[Z]$ be of formal degrees $m_{1}<n_{1}$ and $m_{2}<n_{2}$ respectively. If $q_{1}(a)=q_{2}(b)$ then $q_{1}=q_{2}=r \in R$.

Proof. The statement follows immediately since the $R$-basis $a^{i}, i>0$ and $b^{j}, j>0$ are linearly independent.

Corollary 3.12. Let $R$ be an object of $\mathscr{R} \mathscr{A}_{K}$ and $p \in R[X]$ separable and monic. Let $R[a]=R[X] /\langle p\rangle$ and $\varphi: R \rightarrow R[a]$ the canonical morphism. Let $R[b, c]=R[X, Y] /\langle p(X), p(Y)\rangle$. The commuting diagram 


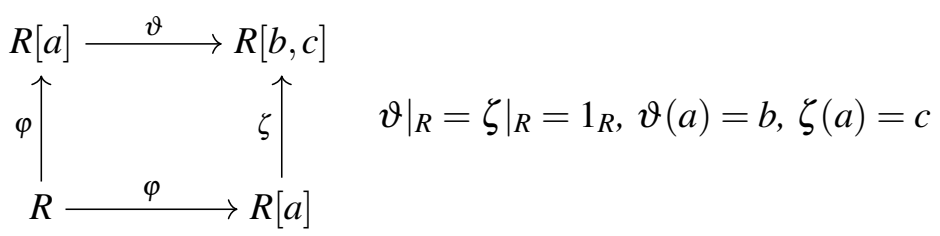

is a pushout diagram of $\mathscr{R} \mathscr{A}_{K}$. Moreover, $\varphi$ is the equalizer of $\zeta$ and $\vartheta$.

Proof. Let $R[a] \stackrel{\eta}{\underset{\rho}{\longrightarrow}} B$ be morphisms of $\mathscr{R} \mathscr{A}_{K}$ such that $\eta \varphi=\rho \varphi$. Then for all $r \in R$ we have $\eta(r)=$ $\rho(r)$. Let $\gamma: R[b, c] \rightarrow B$ be the homomorphism such that $\gamma(r)=\eta(r)=\rho(r)$ for all $r \in R$ while $\gamma(b)=$ $\eta(a), \gamma(c)=\rho(a)$. Then $\gamma$ is the unique map such that $\gamma \vartheta=\eta$ and $\gamma \zeta=\rho$.

Let $A$ be an object of $\mathscr{R} \mathscr{A}_{K}$ and let $\varepsilon: A \rightarrow R[a]$ be a map such that $\zeta \varepsilon=\vartheta \varepsilon$. By Lemma 3.11 if for some $f \in R[a]$ one has $\zeta(f)=\vartheta(f)$ then $f \in R$ (i.e. $f$ is of degree 0 as a polynomial in $a$ over $R$ ). Thus $\varepsilon(A) \subset R$ and we can factor $\varepsilon$ uniquely (since $\varphi$ is injective) as $\varepsilon=\varphi \mu$ with $\mu: A \rightarrow R$.

Let $\{\varphi: R \rightarrow R[a]\}$ be a singleton elementary cocover. Since one can form the pushout of $\varphi$ with itself, the compatibility condition on a singleton family $\{s \in \mathbf{F}(R[a])\}$ can be simplified as: Let $R \stackrel{\varphi}{\longrightarrow} R[a] \underset{\vartheta}{\stackrel{\eta}{\longrightarrow}} A$ be a pushout diagram. A family $\{s \in \mathbf{F}(R[a])\}$ is compatible if and only if $s \vartheta=s \eta$.

Corollary 3.13. The coverage $\mathbf{J}$ is subcanonical, i.e. all representable presheaves in $\mathbf{S e t}^{\mathscr{R} \mathscr{A}_{K}}$ are sheaves on $\left(\mathscr{R} \mathscr{A}_{K}^{o p}, \mathbf{J}\right)$.

\section{The algebraically closed field extension}

We define the presheaf $\mathbf{F}: \mathscr{R} \mathscr{A}_{K} \rightarrow$ Set to be the forgetful functor. That is, for an object $A$ of $\mathscr{R} \mathscr{A}_{K}$, $\mathbf{F}(A)=A$ and for a morphism $\varphi: A \rightarrow C$ of $\mathscr{R} \mathscr{A}_{K}, \mathbf{F}(\varphi)=\varphi$.

Lemma 4.1. $\mathbf{F}$ is a sheaf of sets on the site $\left(\mathscr{R} \mathscr{A}_{K}^{o p}, \mathbf{J}\right)$

Proof. By case analysis on the clauses of Definition 3.7

(i.) Let $\left\{R \stackrel{\varphi_{i}}{\rightarrow} R /\left\langle 1-e_{i}\right\rangle\right\}_{i \in I} \in \mathbf{J}^{o p}(R)$, where $\left(e_{i}\right)_{i \in I}$ is fundamental system of orthogonal idempotents of $R$. The presheaf $\mathbf{F}$ has the property $\mathbf{F}(0)=1$. By Lemma 3.9 a family $\left\{a_{i} \in R /\left\langle 1-e_{i}\right\rangle\right\}_{i \in I}$ is a compatible family. By the isomorphism $R \stackrel{\left(\varphi_{i}\right)_{i \in I}}{\longrightarrow} \prod_{i \in I} R /\left\langle 1-e_{i}\right\rangle$ the element $a=\left(a_{i}\right)_{i \in I} \in R$ is the unique element such that $\varphi_{i}(a)=a_{i}$.

(ii.) Let $\{R \stackrel{\varphi}{\rightarrow} R[a]\} \in \mathbf{J}^{o p}(R)$ where $R[a]=R[X] /\langle p\rangle$ with $p \in R[X]$ monic, non-constant and separable polynomial. Let $\{r \in R[a]\}$ be a compatible family. Let $R \stackrel{\varphi}{\longrightarrow} R[a] \underset{\zeta}{\stackrel{\vartheta}{\longrightarrow}} R[b, c]$ be the pushout diagram of Corollary 3.12. Compatibility then implies $\vartheta(r)=\zeta(r)$ which by the same Corollary is true only if the element $r$ is in $R$. We then have that $r$ is the unique element restricting to itself along the embedding $\varphi$.

We fix a field $K$ of characteristic 0 . Let $\mathscr{L}[F,+,$.$] be a language with basic type F$ and function symbols,.$+: F \times F \rightarrow F$. We extend $\mathscr{L}[F,+,$.$] by adding a constant symbol of type F$ for each element $a \in K$, to obtain $\mathscr{L}[F,+, .]_{K}$. Define $\operatorname{Diag}(K)$ as : if $\phi$ is an atomic $\mathscr{L}[F,+, .]_{K}$-formula or the negation 
of one such that $K=\phi\left(a_{1}, \ldots, a_{n}\right)$ then $\phi\left(a_{1}, \ldots, a_{n}\right) \in \operatorname{Diag}(K)$. The theory $T$ equips the type $F$ with axioms of the geometric theory of algebraically closed field containing $K$

Definition 4.2. The theory $T$ has the following sentences (with all the variables having the type $F$ ).

1. $\operatorname{Diag}(K)$.

2. The axioms of a commutative group: (a) $\forall x[0+x=x+0=x]$ (b) $\forall x \forall y \forall z[x+(y+z)=(x+y)+z]$ (c) $\forall x \exists y[x+y=0]$ (d) $\forall x \forall y[x+y=y+x]$

3. The axioms of a commutative ring: (a) $\forall x[x 1=x]$ (b) $\forall x[x 0=0]$ (c) $\forall x \forall y[x y=y x]$

(d) $\forall x \forall y \forall z[x(y z)=(x y) z]$ (e) $\forall x \forall y \forall z[x(y+z)=x y+x z]$

4. The field axioms: (a) $1 \neq 0$. (b) $\forall x[x=0 \vee \exists y[x y=1]]$.

5. The axiom schema for algebraic closure: $\forall a_{1} \ldots \forall a_{n} \exists x\left[x^{n}+\sum_{i=1}^{n} x^{n-i} a_{i}=0\right]$.

6. $F$ is algebraic over $K: \forall x\left[\bigvee_{p \in K[Y]} p(x)=0\right]$.

With these axioms the type $F$ becomes the type of an algebraically closed field containing $K$. We proceed to show that with the interpretation of the type $F$ by the object $\mathbf{F}$ the topos $\operatorname{Sh}\left(\mathscr{R} \mathscr{A}_{K}^{o p}, \mathbf{J}\right)$ is a model of $T$, i.e. $\mathbf{F}$ is a model, in Kripke-Joyal semantics, of an algebraically closed field containing of $K$. First note that since there is a unique map $K \rightarrow C$ for any object $C$ of $\mathscr{R} \mathscr{A}_{K}$, an element $a \in K$ gives rise to a unique map $\mathbf{1} \stackrel{a}{\rightarrow} \mathbf{F}$, that is the map $* \mapsto a \in \mathbf{F}(K)$. Every constant $a \in K$ of the language is then interpreted by the corresponding unique arrow $\mathbf{1} \stackrel{a}{\rightarrow} \mathbf{F}$. (we use the same symbol for constants and their interpretation to avoid cumbersome notation). That $\mathbf{F}$ satisfies $\operatorname{Diag}(K)$ then follows directly.

Lemma 4.3. $\mathbf{F}$ is a ring object.

Proof. For an object $C$ of $\mathscr{R} \mathscr{A}_{K}$ the object $\mathbf{F}(C)$ is a commutative ring.

Lemma 4.4. $\mathbf{F}$ is a field.

Proof. For any object $R$ of $\mathscr{R} \mathscr{A}_{K}$ one has $R \Vdash 1 \neq 0$ since for any $R \stackrel{\varphi}{\rightarrow} C$ such that $C \Vdash 1=0$ one has that $C$ is trivial and thus $C \Vdash \perp$. Next we show that for variables $x$ and $y$ of type $\mathbf{F}$ and any object $R$ of $\mathscr{R} \mathscr{A}_{K}^{o p}$ we have $R \Vdash \forall x[x=0 \vee \exists y[x y=1]]$. Let $\varphi: A \rightarrow R$ be a morphism of $\mathscr{R}_{K}^{o p}$ and let $a \in A$. We need to show that $A \Vdash a=0 \vee \exists y[y a=1]$. The element $e=a a^{*}$ is an idempotent and we have a cover $\left\{\varphi_{1}\right.$ : $\left.A /\langle e\rangle \rightarrow A, \varphi_{2}: A /\langle 1-e\rangle \rightarrow A\right\} \in \mathbf{J}^{*}(A)$ with $A /\langle e\rangle \Vdash a \varphi_{1}=0$ and $A /\langle 1-e\rangle \Vdash\left(a \varphi_{2}\right)\left(a^{*} \varphi_{2}\right)=e \varphi_{2}=1$. Hence by $\exists$ we have $A /\langle 1-e\rangle \Vdash \exists y\left[\left(a \varphi_{2}\right) y=1\right]$ and by $\vee \vee, A /\langle 1-e\rangle \Vdash a \varphi_{2}=0 \vee \exists y\left[\left(a \varphi_{2}\right) y=1\right]$. Similarly, $A /\langle e\rangle \Vdash a \varphi_{1}=0 \vee \exists y\left[\left(a \varphi_{1}\right) y=1\right]$. By $\forall$ we get $R \Vdash \forall x[x=0 \vee \exists y[x y=1]]$.

To show that $A \Vdash \forall a_{1} \ldots \forall a_{n} \exists x\left[x^{n}+\sum_{i=1}^{n} x^{n-i} a_{i}=0\right]$ for every $n$, we need to be able to extend an algebra $R$ of $\mathscr{R} \mathscr{A}_{K}$ with the appropriate roots. We need the following lemma.

Lemma 4.5. Let $L$ be a field and $f \in L[X]$ a monic polynomial. Let $g=\left\langle f, f^{\prime}\right\rangle$, where $f^{\prime}$ is the derivative of $f$. Writing $f=h g$ we have that $h$ is separable. We call $h$ the separable associate of $f$.

Proof. Let $a$ be the gcd of $h$ and $h^{\prime}$. We have $h=l_{1} a$. Let $d$ be the gcd of $a$ and $a^{\prime}$. We have $a=l_{2} d$ and $a^{\prime}=m_{2} d$, with $l_{2}$ and $m_{2}$ coprime.

The polynomial $a$ divides $h^{\prime}=l_{1} a^{\prime}+l_{1}^{\prime} a$ and hence that $a=l_{2} d$ divides $l_{1} a^{\prime}=l_{1} m_{2} d$. It follows that $l_{2}$ divides $l_{1} m_{2}$ and since $l_{2}$ and $m_{2}$ are coprime, that $l_{2}$ divides $l_{1}$.

Also, if $a^{n}$ divides $p$ then $p=q a^{n}$ and $p^{\prime}=q^{\prime} a^{n}+n q a^{\prime} a^{n-1}$. Hence $d a^{n-1}$ divides $p^{\prime}$. Since $l_{2}$ divides $l_{1}$, this implies that $a^{n}=l_{2} d a^{n-1}$ divides $l_{1} p^{\prime}$. So $a^{n+1}$ divides $a l_{1} p^{\prime}=h p^{\prime}$. 
Since $a$ divides $f$ and $f^{\prime}, a$ divides $g$. We show that $a^{n}$ divides $g$ for all $n$ by induction on $n$. If $a^{n}$ divides $g$ we have just seen that $a^{n+1}$ divides $g^{\prime} h$. Also $a^{n+1}$ divides $h^{\prime} g$ since $a$ divides $h^{\prime}$. So $a^{n+1}$ divides $g^{\prime} h+h^{\prime} g=f^{\prime}$. On the other hand, $a^{n+1}$ divides $f=h g=l_{1} a g$. So $a^{n+1}$ divides $g$ which is the gcd of $f$ and $f^{\prime}$. This implies that $a$ is a unit.

Since $\mathbf{F}$ is a field, the previous lemma holds for polynomials over $\mathbf{F}$. This means that for all objects $R$ of $\mathscr{R} \mathscr{A}_{K}^{o p}$ we have $R \Vdash$ Lemma 4.5. Thus we have the following Corollary.

Corollary 4.6. Let $R$ be an object of $\mathscr{R} \mathscr{A}_{K}$ and let $f$ be a monic polynomial of degree $n$ in $R[X]$ and $f^{\prime}$ its derivative. There is a cocover $\left\{\varphi_{i}: R \rightarrow R_{i}\right\}_{i \in I} \in \mathbf{J}^{* o p}(R)$ and for each $R_{i}$ we have $h, g, q, r, s \in R_{i}[X]$ such that $\varphi_{i}(f)=h g, \varphi_{i}\left(f^{\prime}\right)=q g$ and $r h+s q=1$. Moreover, $h$ is monic and separable.

Note that in characteristic 0 , if $f$ is monic and non-constant the separable associate of $f$ is nonconstant.

Lemma 4.7. The field object $\mathbf{F} \in \operatorname{Sh}\left(\mathscr{R}_{\mathscr{A}_{K}^{o p}}, \mathbf{J}\right)$ is algebraically closed.

Proof. We prove that for all $n>0$ and all $\left(a_{1}, \ldots, a_{n}\right) \in \mathbf{F}^{n}(R)=R^{n}$, one has $R \Vdash \exists x\left[x^{n}+\sum_{i=1}^{n} x^{n-i} a_{i}=0\right]$. Let $f=x^{n}+\sum_{i=1}^{n} x^{n-i} a_{i}$. By Corollary 4.6 we have a cover $\left\{\vartheta_{j}: R_{j} \rightarrow R\right\}_{j \in I} \in \mathbf{J}^{*}(R)$ such that in each $R_{j}$ we have $g=\left\langle f \vartheta_{j}, f^{\prime} \vartheta_{j}\right\rangle$ and $f \vartheta_{j}=h g$ with $h \in R_{j}[X]$ monic and separable. Note that if $\operatorname{deg} f \geq 1, h$ is non-constant. For each $R_{j}$ we have a singleton cover $\left\{\varphi: R_{j}[b] \rightarrow R_{j} \mid R_{j}[b]=R_{j}[X] /\langle h\rangle\right\} \in \mathbf{J}^{*}\left(R_{j}\right)$. That is, we have $R_{j}[b] \Vdash b^{n}+\sum_{i=1}^{n} b^{n-1}\left(a_{i} \vartheta_{j} \varphi\right)=0$. By $\exists \exists$ we get $R_{j}[b] \Vdash \exists x\left[x^{n}+\sum_{i=1}^{n} x^{n-1}\left(a_{i} \vartheta_{j} \varphi\right)=0\right]$ and by LC we have $R_{j} \Vdash \exists x\left[x^{n}+\sum_{i=1}^{n} x^{n-1}\left(a_{i} \vartheta_{j}\right)=0\right]$. Since this is true for each $R_{j}, j \in J$ we have by LC $R \Vdash \exists x\left[x^{n}+\sum_{i=1}^{n} x^{n-1} a_{i}=0\right]$.

Lemma 4.8. $\mathbf{F}$ is algebraic over $K$.

Proof. We will show that for any object $R$ of $\mathscr{R}_{\mathscr{A}_{K}}$ and element $r \in R$ one has $R \Vdash \bigvee_{p \in K[X]} p(r)=0$. Since $R$ is a finitely presented $K$-algebra we have that $R$ is a finite integral extension of a polynomial ring $K\left[Y_{1}, \ldots, Y_{n}\right] \subset R$ where $Y_{1}, . ., Y_{n}$ are elements of $R$ algebraically independent over $K$ and that $R$ has Krull dimension $n$ [9, Ch 13, Theorem 5.4]. Since $R$ is zero-dimensional (i.e. has Krull dimension 0) we have $n=0$ and $R$ is integral over $K$, i.e. any element $r \in R$ is the zero of some monic polynomial over $K$.

\section{Constant sheaves, natural numbers, and power series}

Here we describe the object of natural numbers in the topos $\operatorname{Sh}\left(\mathscr{R} \mathscr{A}_{K}^{o p}, \mathbf{J}\right)$ and the object of power series over the field $\mathbf{F}$. This will be used in section 6 to show that the axiom of dependent choice does not hold when the base field $K$ is the rationals and later in the example of Newton-Puiseux theorem (section77).

Let $\mathbf{P}: \mathscr{R} \mathscr{A}_{K} \rightarrow$ Set be a constant presheaf associating to each object $A$ of $\mathscr{R} \mathscr{A}_{K}$ a discrete set $B$. That is, $\mathbf{P}(A)=B$ and $\mathbf{P}(A \stackrel{\varphi}{\rightarrow} R)=1_{B}$ for all objects $A$ and all morphism $\varphi$ of $\mathscr{R} \mathscr{A}_{K}$. Let $\widetilde{\mathbf{P}}: \mathscr{R} \mathscr{A}_{K} \rightarrow$ Set be the presheaf such that $\widetilde{\mathbf{P}}(A)$ is the set of elements of the form $\left\{\left(e_{i}, b_{i}\right)\right\}_{i \in I}$ where $\left(e_{i}\right)_{i \in I}$ is a fundamental system of orthogonal idempotents of $A$ and for each $i, b_{i} \in B$. We express such an element as a formal sum $\sum_{i \in I} e_{i} b_{i}$. Let $\varphi: A \rightarrow R$ be a morphism of $\mathscr{R} \mathscr{A}_{K}$, the restriction of $\sum_{i \in I} e_{i} b_{i} \in \widetilde{\mathbf{P}}(A)$ along $\varphi$ is given by $\left(\sum_{i \in I} e_{i} b_{i}\right) \varphi=\sum_{i \in I} \varphi\left(e_{i}\right) b_{i} \in \widetilde{\mathbf{P}}(R)$. In particular with canonical morphisms $\varphi_{i}: A \rightarrow A /\left\langle 1-e_{i}\right\rangle$, one has for any $j \in I$ that $\left(\sum_{i \in I} e_{i} b_{i}\right) \varphi_{j}=b_{j} \in \widetilde{\mathbf{P}}\left(A /\left\langle 1-e_{j}\right\rangle\right)$. Two elements $\sum_{i \in I} e_{i} b_{i} \in \widetilde{\mathbf{P}}(A)$ and $\sum_{j \in J} d_{j} c_{j} \in \widetilde{\mathbf{P}}(A)$ are equal if and only if $\forall i \in I, j \in J\left[b_{i} \neq c_{j} \Rightarrow e_{i} d_{j}=0\right]$.

To prove that $\widetilde{\mathbf{P}}$ is a sheaf we will need the following lemmas. 
Lemma 5.1. Let $R$ be a regular ring and let $\left(e_{i}\right)_{i \in I}$ be a fundamental system of orthogonal idempotents of $R$. Let $R_{i}=R /\left\langle 1-e_{i}\right\rangle$ and $\left(\left[d_{j}\right]\right)_{j \in J_{i}}$ be a fundamental system of orthogonal idempotents of $R_{i}$, where $\left[d_{j}\right]=d_{j}+\left\langle 1-e_{i}\right\rangle$. The family $\left(e_{i} d_{j}\right)_{i \in I, j \in J_{i}}$ is a fundamental system of orthogonal idempotents of $R$.

Proof. In $R$ one has $\sum_{j \in J_{i}} e_{i} d_{j}=e_{i} \sum_{j \in J_{i}} d_{j}=e_{i}\left(1+\left\langle 1-e_{i}\right\rangle\right)=e_{i}$. Hence, $\sum_{i \in I, j \in J_{i}} e_{i} d_{j}=\sum_{i \in I} e_{i}=1$. For some $i \in I$ and $t, k \in J_{i}$ we have $\left(e_{i} d_{t}\right)\left(e_{i} d_{k}\right)=e_{i}\left(0+\left\langle 1-e_{i}\right\rangle\right)=0$ in $R$. Thus for $i, \ell \in I, j \in J_{i}$ and $s \in J_{\ell}$ one has $i \neq \ell \vee j \neq s \Rightarrow\left(e_{i} d_{j}\right)\left(e_{\ell} d_{s}\right)=0$.

Lemma 5.2. Let $R$ be a regular ring, $f \in R[Z]$ a polynomial of formal degree $n$ and $p \in R[Z]$ a monic polynomial of degree $m>n$. If in $R[X, Y]$ one has $f(Y)(1-f(X))=0 \bmod \langle p(X), p(Y)\rangle$ then $f=e \in R$ with e an idempotent.

Proof. Let $f(Z)=\sum_{i=0}^{n} r_{i} Z^{i}$. By the assumption, for some $q, g \in R[X, Y]$

$$
f(Y)(1-f(X))=\sum_{i=0}^{n} r_{i}\left(1-\sum_{j=0}^{n} r_{j} X^{j}\right) Y^{i}=q p(X)+g p(Y)
$$

One has $\sum_{i=0}^{n} r_{i}\left(1-\sum_{j=0}^{n} r_{j} X^{j}\right) Y^{i}=g(X, Y) p(Y) \bmod \langle p(X)\rangle$. Since $p(Y)$ is monic of $Y$-degree greater than $n$, one has that $r_{i}\left(1-\sum_{j=0}^{n} r_{j} X^{j}\right)=0 \bmod \langle p(X)\rangle$ for all $0 \leq i \leq n$. But this means that $r_{i} r_{n} X^{n}+$ $r_{i} r_{n-1} X^{n-1}+\ldots+r_{i} r_{0}-r_{i}$ is divisible by $p(X)$ for all $0 \leq i \leq n$ which because $p(X)$ is monic of degree $m>n$ implies that all coefficients are equal to 0 . In particular, for $1 \leq i \leq n$ one gets that $r_{i}^{2}=0$ and hence $r_{i}=0$ since $R$ is reduced. For $i=0$ we have $r_{0} r_{0}-r_{0}=0$ and thus $r_{0}$ is an idempotent of $R$.

Lemma 5.3. The presheaf $\widetilde{\mathbf{P}}$ described above is a sheaf on $\left(\mathscr{R} \mathscr{A}_{K}^{o p}, \mathbf{J}\right)$.

Proof. By case analysis on Definition 3.7

(i.) Let $\left\{R \stackrel{\varphi_{i}}{\rightarrow} R /\left\langle 1-e_{i}\right\rangle\right\}_{i \in I} \in \mathbf{J}^{o p}(R)$ where $\left(e_{i}\right)_{i \in I}$ be a fundamental system of orthogonal idempotents of an object $R$. Let $R /\left\langle 1-e_{i}\right\rangle=R_{i}$. Since $\widetilde{\mathbf{P}}(0)=1$ by Lemma 3.9 any set $\left\{s_{i} \in\right.$ $\left.\widetilde{\mathbf{P}}\left(R_{i}\right)\right\}_{i \in I}$ is compatible. For each $i$, Let $s_{i}=\sum_{j \in J_{i}}\left[d_{j}\right] b_{j}$. By Lemma 5.1 we have an element $s=\sum_{i \in I, j \in J_{i}}\left(e_{i} d_{j}\right) b_{j} \in \widetilde{\mathbf{P}}(R)$ the restriction of which along $\varphi_{i}$ is the element $\sum_{j \in J_{i}}\left[d_{j}\right] b_{j} \in \widetilde{\mathbf{P}}\left(R_{i}\right)$.

It remains to show that this is the only such element. Let there be an element $\sum_{\ell \in L} c_{\ell} a_{\ell} \in \widetilde{\mathbf{P}}(R)$ that restricts to $u_{i}=s_{i}$ along $\varphi_{i}$. We have $u_{i}=\sum_{\ell \in L}\left[c_{\ell}\right] a_{\ell}$. One has that for any $j \in J_{i}$ and $\ell \in L$, $b_{j} \neq a_{\ell} \Rightarrow\left[c_{\ell} d_{j}\right]=0$ in $R_{i}$, hence, in $R$ one has $b_{j} \neq a_{\ell} \Rightarrow c_{\ell} d_{j}=r\left(1-e_{i}\right)$. Multiplying both sides of $c_{\ell} d_{j}=r\left(1-e_{i}\right)$ by $e_{i}$ we get $b_{j} \neq a_{\ell} \Rightarrow c_{\ell}\left(e_{i} d_{j}\right)=0$. Thus proving $s=\sum_{\ell \in L} c_{\ell} a_{\ell}$.

(ii.) Let $\{\varphi: R \rightarrow R[a]=R[X] /\langle p\rangle\} \in \mathbf{J}^{o p}(R)$ where $p \in R[X]$ is monic non-constant and separable. Let the singleton $\left\{s=\sum_{i \in I} e_{i} b_{i} \in \widetilde{\mathbf{P}}(R[a])\right\}$ be compatible. We can assume w.l.o.g. that $\forall i, j \in I\left[i \neq j \Rightarrow b_{i} \neq b_{j}\right]$ since if $b_{k}=b_{\ell}$ one has that $\left(e_{k}+e_{\ell}\right) b_{l}+\sum_{j \in I}^{j \neq \ell, j \neq k} e_{j} b_{j}=s$. (Note that an idempotent $e_{i}$ of $R[a]$ is a polynomial $e_{i}(a)$ in $a$ of formal degree less than $\operatorname{deg} p$ ). Let $R[c, d]=$ $R[X, Y] /\langle p(X), p(Y)\rangle$, by Corollary 3.12 , one has a pushout diagram $R \stackrel{\varphi}{\longrightarrow} R[a] \stackrel{\zeta}{\underset{\vartheta}{\longrightarrow}} R[c, d]$ where $\left.\zeta\right|_{R}=\left.\vartheta\right|_{R}=1_{R}, \zeta(a)=d$ and $\vartheta(a)=c$. That the singleton $\{s\}$ is compatible then means $s \vartheta=\sum_{i \in I} e_{i}(c) b_{i}=s \zeta=\sum_{i \in I} e_{i}(d) b_{i}$, i.e. $\forall i, j \in I\left[b_{i} \neq b_{j} \Rightarrow e_{i}(c) e_{j}(d)=0\right]$. By the assumption that $b_{i} \neq b_{j}$ whenever $i \neq j$ we have in $R[c, d]$ that $e_{j}(d) e_{i}(c)=0$ for any $i \neq j \in$ I. Thus $e_{j}(d) \sum_{i \neq j} e_{i}(c)=e_{j}(d)\left(1-e_{j}(c)\right)=0$, i.e. in $R[X, Y]$ one has $e_{j}(Y)\left(1-e_{j}(X)\right)=0$ $\bmod \langle p(X), p(Y)\rangle$. By Lemma 5.2 we have that $e_{j}(X)=e_{j}(Y)=e \in R$. We have thus shown $s$ is 
equal to $\sum_{j \in J} d_{j} b_{j} \in \widetilde{\mathbf{P}}(R[a])$ such that $d_{j} \in R$ for $j \in J$. That is $\sum_{j \in J} d_{j} b_{j} \in \widetilde{\mathbf{P}}(R)$. Thus we have found a unique (since $\widetilde{\mathbf{P}}(\varphi)$ is injective) element in $\widetilde{\mathbf{P}}(R)$ restricting to $s$ along $\varphi$.

Lemma 5.4. Let $\mathbf{P}$ and $\widetilde{\mathbf{P}}$ be as described above. Let $\Gamma: \mathbf{P} \rightarrow \widetilde{\mathbf{P}}$ be the presheaf morphism such that $\Gamma_{R}(b)=b \in \widetilde{\mathbf{P}}(R)$ for any object $R$ and $b \in B$. If $\mathbf{E}$ is a sheaf and $\Lambda: \mathbf{P} \rightarrow \mathbf{E}$ is a morphism of presheaves, then there exist a unique sheaf morphism $\Delta: \widetilde{\mathbf{P}} \rightarrow \mathbf{E}$ such that the following diagram, of $\mathbf{S e t}^{\mathscr{R} \mathscr{A}_{K}}$, com-

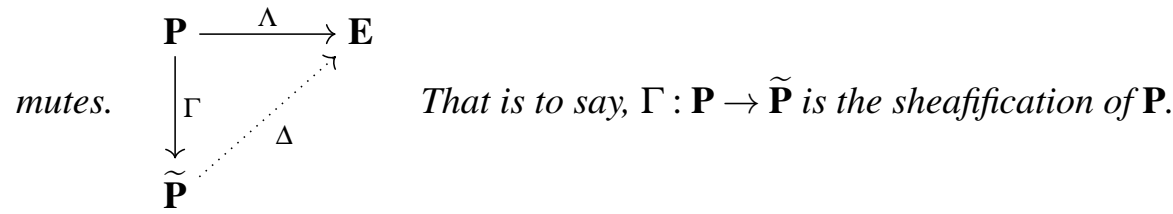

Proof. Let $a=\sum_{i \in I} e_{i} b_{i} \in \widetilde{\mathbf{P}}(A)$ and let $A_{i}=A /\left\langle 1-e_{i}\right\rangle$ with canonical morphisms $\varphi_{i}: A \rightarrow A_{i}$.

Let $\mathbf{E}$ and $\Lambda$ be as in the statement of the lemma. If there exist a sheaf morphism $\Delta: \widetilde{\mathbf{P}} \rightarrow \mathbf{E}$, then $\Delta$ being a natural transformation forces us to have for all $i \in I, \mathbf{E}\left(\varphi_{i}\right) \Delta_{A}=\Delta_{A_{i}} \widetilde{\mathbf{P}}\left(\varphi_{i}\right)$. By Lemma 3.10, we know that the map $d \in \mathbf{E}(A) \mapsto\left(\mathbf{E}\left(\varphi_{i}\right) d \in \mathbf{E}\left(A_{i}\right)\right)_{i \in I}$ is an isomorphism. Thus it must be that $\Delta_{A}(a)=\left(\Delta_{A_{i}} \widetilde{\mathbf{P}}\left(\varphi_{i}\right)(a)\right)_{i \in I}=\left(\Delta_{A_{i}}\left(b_{i}\right)\right)_{i \in I}$. But $\Delta_{A_{i}}\left(b_{i}\right)=\Delta_{A_{i}} \Gamma_{A_{i}}\left(b_{i}\right)$. To have $\Delta \Gamma=\Lambda$ we must have $\Delta_{A_{i}}\left(b_{i}\right)=\Lambda_{A_{i}}\left(b_{i}\right)$. Hence, we are forced to have $\Delta_{A}(a)=\left(\Lambda_{A_{i}}\left(b_{i}\right)\right)_{i \in I}$. Note that $\Delta$ is unique since its value $\Delta_{A}(a)$ at any $A$ and $a$ is forced by the commuting diagram above.

The constant presheaf of natural numbers $\mathbf{N}$ is the natural numbers object in Set $^{\mathscr{R} \mathscr{A}}{ }_{K}$. We associate to $\mathbf{N}$ a sheaf $\widetilde{\mathbf{N}}$ as described above. From Lemma 5.4 one can easily show that $\widetilde{\mathbf{N}}$ satisfy the axioms of a natural numbers object in $\operatorname{Sh}\left(\mathscr{R} \mathscr{A}_{K}^{o p}, \mathbf{J}\right)$.

Definition 5.5. Let $\mathbf{F}[[X]]$ be the presheaf mapping each object $R$ of $\mathscr{R}_{\mathscr{A}_{K}}$ to $\mathbf{F}[[X]](R)=R[[X]]=R^{\mathbb{N}}$ with the obvious restriction maps.

Lemma 5.6. $\mathbf{F}[[X]]$ is a sheaf.

Proof. The proof is immediate as a corollary of Lemma 4.1.

Lemma 5.7. The sheaf $\mathbf{F}[[X]]$ is naturally isomorphic to the sheaf $\mathbf{F}^{\widetilde{\mathbf{N}}}$.

Proof. Let $C$ be an object of $\mathscr{R} \mathscr{A}_{K}^{o p}$. Since $\mathbf{F}^{\widetilde{\mathbf{N}}}(C) \cong \mathbf{y}_{C} \times \widetilde{\mathbf{N}} \rightarrow \mathbf{F}$, an element $\alpha_{C} \in \mathbf{F}^{\widetilde{\mathbf{N}}}(C)$ is a family of elements of the form $\alpha_{C, D}: \mathbf{y}_{C}(D) \times \widetilde{\mathbf{N}}(D) \rightarrow \mathbf{F}(D)$ where $D$ is an object of $\mathscr{R} \mathscr{A}_{K}^{o p}$. Define $\Theta: \mathbf{F}^{\widetilde{\mathbf{N}}} \rightarrow$ $\mathbf{F}[[X]]$ as $(\Theta \alpha)_{C}(n)=\alpha_{C, C}\left(1_{C}, n\right)$. Define $\Lambda: \mathbf{F}[[X]] \rightarrow \mathbf{F}^{\widetilde{\mathbf{N}}}$ as

$$
(\Lambda \beta)_{C, D}\left(C \stackrel{\varphi}{\rightarrow} D, \sum_{i \in I} e_{i} n_{i}\right)=\left(\vartheta_{i} \varphi\left(\beta_{C}\left(n_{i}\right)\right)\right)_{i \in I} \in \mathbf{F}(D)
$$

where $D \stackrel{\vartheta_{i}}{\rightarrow} D /\left\langle 1-e_{i}\right\rangle$ is the canonical morphism. Note that by Lemma 3.10 one indeed has that $\left(\vartheta_{i} \varphi\left(\beta_{C}\left(n_{i}\right)\right)\right)_{i \in I} \in \prod_{i \in I} \mathbf{F}\left(D_{i}\right) \cong \mathbf{F}(D)$. One can easily verify that $\Theta$ and $\Lambda$ are natural. It remains to show the isomorphism. One one hand we have

$$
\begin{aligned}
(\Lambda \Theta \alpha)_{C, D}\left(\varphi, \sum_{i \in I} e_{i} n_{i}\right) & =\left(\vartheta_{i} \varphi\left((\Theta \alpha)_{C}\left(n_{i}\right)\right)\right)_{i \in I}=\left(\vartheta_{i} \varphi\left(\alpha_{C, C}\left(1_{C}, n_{i}\right)\right)\right)_{i \in I} \\
& =\left(\left(\alpha_{C, D_{i}}\left(\vartheta_{i} \varphi, n_{i}\right)\right)\right)_{i \in I}=\alpha_{C, D}\left(\varphi, \sum_{i \in I} e_{i} n_{i}\right)
\end{aligned}
$$

Thus showing $\Lambda \Theta=1_{\mathbf{F}^{\tilde{\mathbf{N}}}}$. On the other hand, $(\Theta \Lambda \beta)_{C}(n)=(\Lambda \beta)_{C, C}\left(1_{C}, n\right)=1_{C} 1_{C}\left(\beta_{C}(n)\right)=\beta_{C}(n)$. Thus $\Theta \Lambda=1_{\mathbf{F}[[X]]}$. 
Lemma 5.8. The power series object $\mathbf{F}[[X]]$ is a ring object.

Proof. A Corollary to Lemma 4.3 .

\section{Choice axioms}

The (external) axiom of choice fails to hold (even in a classical metatheory) in the topos $\operatorname{Sh}\left(\mathscr{R} \mathscr{A}_{K}^{o p}, \mathbf{J}\right)$ whenever the field $K$ is not algebraically closed. To show this we will show that there is an epimorphism in $\operatorname{Sh}\left(\mathscr{R} \mathscr{A}_{K}^{o p}, \mathbf{J}\right)$ with no section.

Fact 6.1. Let $\Theta: \mathbf{P} \rightarrow \mathbf{G}$ be a morphism of sheaves on a site $(\mathscr{C}, \mathbf{J})$. Then $\Theta$ is an epimorphism if for each object $C$ of $\mathscr{C}$ and each element $c \in \mathbf{G}(C)$ there is a cover $S$ of $C$ such that for all $f: D \rightarrow C$ in the cover $S$ the element cf is in the image of $\Theta_{D}$. [10, Ch. 3].

Lemma 6.2. Let $K$ be a field of characteristic 0 not algebraically closed. There is an epimorphism in $\operatorname{Sh}\left(\mathscr{R} \mathscr{A}_{K}^{o p}, \mathbf{J}\right)$ with no section.

Proof. Let $f=X^{n}+\sum_{i=1}^{n} r_{i} X^{n-i}$ be a non-constant polynomial for which no root in $K$ exist. w.l.o.g. we assume $f$ separable. One can construct $\Lambda: \mathbf{F} \rightarrow \mathbf{F}$ defined by $\Lambda_{C}(c)=c^{n}+\sum_{i=1}^{n-1} r_{i} c^{n-i} \in C$. Given $d \in \mathbf{F}(C)$, let $g=X^{n}+\sum_{i=1}^{n-1} r_{i} X^{n-i}-d$. By Corollary 4.6 there is a cover $\left\{C_{\ell} \stackrel{\varphi_{\ell}}{\longrightarrow} C\right\}_{\ell \in L} \in \mathbf{J}^{*}(C)$ with $h_{\ell} \in C_{\ell}[X]$ a separable non-constant polynomial dividing $g$. Let $C_{\ell}\left[x_{\ell}\right]=C_{\ell}[X] /\left\langle h_{\ell}\right\rangle$ one has a singleton cover $\left\{C_{\ell}\left[x_{\ell}\right] \stackrel{\vartheta_{\ell}}{\longrightarrow} C_{\ell}\right\}$ and thus a composite cover $\left\{C_{\ell}\left[x_{\ell}\right] \stackrel{\vartheta_{\ell} \varphi_{\ell}}{\longrightarrow} C\right\}_{\ell \in L} \in \mathbf{J}^{*}(C)$. Since $x_{\ell}$ is a root of $h_{\ell} \mid g$ we have $\Lambda_{C_{\ell}\left[x_{\ell}\right]}\left(x_{\ell}\right)=x_{\ell}^{n}+\sum_{i=1}^{n-1} r_{i} x_{\ell}^{n-i}=d$ or more precisely $\Lambda_{C_{\ell}\left[x_{\ell}\right]}\left(x_{\ell}\right)=d \varphi_{\ell} \vartheta_{\ell}$. Thus, $\Lambda$ is an epimorphism (by Fact 6.1) and it has no section, for if it had a section $\Psi: \mathbf{F} \rightarrow \mathbf{F}$ then one would have $\Psi_{K}\left(-r_{n}\right)=a \in K$ such that $a^{n}+\sum_{i=1}^{n} r_{i} a^{n-i}=0$ which is not true by assumption.

Theorem 6.3. Let $K$ be a field of characteristic 0 not algebraically closed. The axiom of choice fails to hold in the topos $\operatorname{Sh}\left(\mathscr{R} \mathscr{A}_{K}^{o p}, \mathbf{J}\right)$.

We note that in Per Martin-Löf type theory one can show that (see [13])

$$
\left(\prod x \in A\right)\left(\sum y \in B[x]\right) C[x, y] \Rightarrow\left(\sum f \in\left(\prod x \in A\right) B[x]\right)\left(\prod x \in A\right) C[x, f(x)]
$$

As demonstrated in the topos $\operatorname{Sh}\left(\mathscr{R} \mathscr{A}_{K}^{o p}, \mathbf{J}\right)$ we have an example of an intuitionistically valid formula of the form $\forall x \exists y \phi(x, y)$ where no function $f$ exist for which $\exists f \forall x \phi(x, f(x))$ holds.

We demonstrate further that when the base field is $\mathbb{Q}$ the weaker axiom of dependent choice does not hold (internally) in the topos $\operatorname{Sh}\left(\mathscr{R} \mathscr{A}_{\mathbb{Q}}^{o p}, \mathbf{J}\right)$. For a relation $R \subset Y \times Y$ the axiom of dependent choice is stated as

$$
\forall x \exists y R(x, y) \Rightarrow \forall x \exists g \in Y^{N}[g(0)=x \wedge \forall n R(g(n), g(n+1))]
$$

Theorem 6.4. $\operatorname{Sh}\left(\mathscr{R} \mathscr{A}_{\mathbb{Q}}^{o p}, \mathbf{J}\right) \Vdash \neg \mathrm{ADC}$.

Proof. Consider the binary relation on the algebraically closed object $\mathbf{F}$ defined by the characteristic function $\phi(x, y):=y^{2}-x=0$. Assume $C \Vdash \mathrm{ADC}$ for some object $C$ of $\mathscr{R} \mathscr{A}_{K}$. Since $C \Vdash \forall x \exists y\left[y^{2}-x=\right.$

$0]$ we have $C \Vdash \forall x \exists g \in \mathbf{F}^{\widetilde{\mathbf{N}}}\left[g(0)=x \wedge \forall n\left[g(n)^{2}=g(n+1)\right]\right]$. That is for all morphisms $C \stackrel{\zeta}{\rightarrow} A$ of $\mathscr{R} \mathscr{A}_{K}$ and elements $a \in \mathbf{F}(A)$ one has $A \Vdash \exists g \in \mathbf{F}^{\widetilde{\mathbf{N}}}\left[g(0)=a \wedge \forall n\left[g(n)^{2}=g(n+1)\right]\right]$. Taking $a=2$ we have $A \Vdash \exists g \in \mathbf{F}^{\widetilde{\mathbf{N}}}\left[g(0)=2 \wedge \forall n\left[g(n)^{2}=g(n+1)\right]\right]$. Which by $\exists$ implies the existence of a cocover $\left\{\eta_{i}: A \rightarrow A_{i} \mid i \in I\right\}$ and power series $\alpha_{i} \in \mathbf{F}^{\widetilde{\mathbf{N}}}\left(A_{i}\right)$ such that $\left.A_{i} \Vdash \alpha_{i}(0)=2 \wedge \forall n\left[\alpha_{i}(n)^{2}=\alpha_{i}(n+1)\right]\right]$. 
By Lemma 5.7 we have $\mathbf{F}^{\widetilde{\mathbf{N}}}\left(A_{i}\right) \cong A_{i}[[X]]$ and thus the above forcing implies the existence of a series $\alpha_{i}=2+2^{1 / 2}+\ldots+2^{1 / 2^{j}}+\ldots \in A_{i}[[X]]$. But this holds only if $A_{i}$ contains a root of $X^{2^{j}}-2$ for all $j$ which implies $A_{i}$ is trivial as will shortly show after the following remark.

Consider an algebra $R$ over $\mathbb{Q}$. Assume $R$ contains a root of $X^{2^{n}}-2$ for some $n$. Then letting $\mathbb{Q}[x]=\mathbb{Q}[X] /\left\langle X^{2^{n}}-2\right\rangle$, one will have a homomorphism $\xi: \mathbb{Q}[x] \rightarrow R$. By Eisenstein's criterion the polynomial $X^{2^{n}}-2$ is irreducible over $\mathbb{Q}$, making $\mathbb{Q}[x]$ a field of dimension $2^{n}$ and $\xi$ either an injection with a trivial kernel or $\xi=\mathbb{Q}[x] \rightarrow 0$.

Now we continue with the proof. Until now we have shown that for all $i \in I$, the algebra $A_{i}$ contains a root of $X^{2^{j}}-2$ for all $j$. For each $i \in I$, let $A_{i}$ be of dimension $m_{i}$ over $\mathbb{Q}$. We have that $A_{i}$ contains a root of $X^{2^{m_{i}}}-2$ and we have a homomorphism $\mathbb{Q}\left(\sqrt[2^{m_{i}}]{2}\right) \rightarrow A_{i}$ which since $A_{i}$ has dimension $m_{i}<2^{m_{i}}$ means that $A_{i}$ is trivial for all $i \in I$. Hence, $A_{i} \Vdash \perp$ and consequently $C \Vdash \perp$. We have shown that for any object $D$ of $\mathscr{R} \mathscr{A}_{\mathbb{Q}}^{o p}$ if $D \Vdash$ ADC then $D \Vdash \perp$. Hence $\operatorname{Sh}\left(\mathscr{R} \mathscr{A}_{\mathbb{Q}}^{o p}, \mathbf{J}\right) \Vdash \neg$ ADC.

As a consequence we get that the internal axiom of choice does not hold in $\operatorname{Sh}\left(\mathscr{R} \mathscr{A}_{\mathbb{Q}}^{o p}, \mathbf{J}\right)$.

\section{Eliminating the algebraic closure assumption}

Let $K$ be a field of characteristic 0 . We consider a typed language $\mathscr{L}[N, F]_{K}$ of the form described in Section 2 with two basic types $N$ and $F$ and the elements of the field $K$ as its set of constants. Consider a theory $T$ in the language $\mathscr{L}[N, F]_{K}$, such that $T$ has as an axiom every atomic formula or the negation of one valid in the field $K, T$ equips $N$ with the (Peano) axioms of natural numbers and equips $F$ with the axioms of a field containing $K$. If we interpret the types $N$ and $F$ by the objects $\widetilde{\mathbf{N}}$ and $\mathbf{F}$, respectively, in the topos $\operatorname{Sh}\left(\mathscr{R} \mathscr{A}_{K}^{o p}, \mathbf{J}\right)$ then we have, by the results proved earlier, a model of $T$ in $\operatorname{Sh}\left(\mathscr{R} \mathscr{A}_{K}^{o p}, \mathbf{J}\right)$. Let $\mathrm{AlgCl}$ be the axiom schema of algebraic closure with quantification over the type $F$, then one has that $T+\mathrm{AlgCl}$ has a model in $\operatorname{Sh}\left(\mathscr{R} \mathscr{A}_{K}^{o p}, \mathbf{J}\right)$ with the same interpretation. Let $\phi$ be a sentence in the language such that $T+\mathrm{AlgCl} \vdash \phi$ in IHOL deduction system. By soundness [1] one has that $\operatorname{Sh}\left(\mathscr{R} \mathscr{A}_{K}^{o p}, \mathbf{J}\right) \Vdash \phi$, i.e. for all finite dimensional regular algebras $R$ over $K, R \Vdash \phi$ which is then a constructive interpretation of the existence of the algebraic closure of $K$.

This model can be implemented, e.g. in Haskell. In the paper [12] by the authors, an algorithm for computing the Puiseux expansions of an algebraic curve based on this model is given. The statement with the assumption of algebraic closure is:

" Let $K$ be a field of characteristic 0 and $G(X, Y)=Y^{n}+\sum_{i=1}^{n} b_{i}(X) Y^{n-i} \in K[[X]][Y]$ a monic, nonconstant polynomial separable over $K((X))$. Let $F$ be the algebraic closure of $K$, we have a positive integer $m$ and a factorization $G\left(T^{m}, Y\right)=\prod_{i=1}^{n}\left(Y-\alpha_{i}\right)$ with $\alpha_{i} \in F[[T]]$ ”

We can then extract the following computational content

" Let $K$ be a field of characteristic 0 and $G(X, Y)=Y^{n}+\sum_{i=1}^{n} b_{i}(X) Y^{n-i} \in K[[X]][Y]$ a monic, nonconstant polynomial separable over $K((X))$. Then there exist a (von Neumann) regular algebra $R$ over $K$ and a positive integer $m$ such that $G\left(T^{m}, Y\right)=\prod_{i=1}^{n}\left(Y-\alpha_{i}\right)$ with $\alpha_{i} \in R[[T]]$ ”

For example applying the algorithm to $G(X, Y)=Y^{4}-3 Y^{2}+X Y+X^{2} \in \mathbb{Q}[X, Y]$ we get a regular 
algebra $\mathbb{Q}[b, c]$ with $b^{2}-13 / 36=0$ and $c^{2}-3=0$ and a factorization

$$
\begin{aligned}
& G(X, Y)= \\
& \left(Y+\left(-b-\frac{1}{6}\right) X+\left(-\frac{31}{351} b-\frac{7}{162}\right) X^{3}+\left(-\frac{1415}{41067} b-\frac{29}{1458}\right) X^{5}+\ldots\right) \\
& \left(Y+\left(b-\frac{1}{6}\right) X+\left(\frac{31}{351} b-\frac{7}{162}\right) X^{3}+\left(\frac{1415}{41067} b-\frac{29}{1458}\right) X^{5}+\ldots\right) \\
& \left(Y-c+\frac{1}{6} X+\frac{5}{72} c X^{2}+\frac{7}{162} X^{3}+\frac{185}{10368} c X^{4}+\frac{29}{1458} X^{5}+\ldots\right) \\
& \left(Y+c+\frac{1}{6} X-\frac{5}{72} c X^{2}+\frac{7}{162} X^{3}-\frac{185}{10368} c X^{4}+\frac{29}{1458} X^{5}+\ldots\right)
\end{aligned}
$$

Another example of a possible application of this model is as follows: suppose one want to show that "For discrete field $K$, if $f \in K[X, Y]$ is smooth, i.e. $1 \in\left\langle f, f_{x}, f_{Y}\right\rangle$, then $K[X, Y] /\langle f\rangle$ is a Prüfer ring. "

To prove that a ring is Prüfer one needs to prove that it is arithmetical, that is $\forall x, y \exists u, v, w[y u=$ $v x \wedge y w=(1-u) x]$. Proving that $K[X, Y] /\langle f\rangle$ is arithmetical is easier in the case where $K$ is algebraically closed [3]. Let $\mathbf{F}$ be the algebraic closure of $K$ in $\operatorname{Sh}\left(\mathscr{R} \mathscr{A}_{K}^{o p}, \mathbf{J}\right)$. Now $\mathbf{F}[X, Y] /\langle f\rangle$ being arithmetical amounts to having a solution $u, v$, and $w$ to a linear system $y u=v x, y w=(1-u) x$. Having obtained such solution, by Rouché-Capelli-Fontené theorem we can conclude that the system have a solution in $K[X, Y] /\langle f\rangle$.

\section{The logic of $\operatorname{Sh}\left(\mathscr{R} \mathscr{A}_{K}^{o p}, \mathbf{J}\right)$}

In this section we will demonstrate that in a classical metatheory one can show that the topos $\operatorname{Sh}\left(\mathscr{R} \mathscr{A}_{K}^{o p}, \mathbf{J}\right)$ is boolean. In fact we will show that, in a classical metatheory, the boolean algebra structure of the subobject classifier is the one specified by the boolean algebra of idempotents of the algebras in $\mathscr{R} \mathscr{A}_{K}$. Except for Theorem 8.8 the reasoning in this section is classical. Recall that the idempotents of a commutative ring form a boolean algebra with the meaning of the logical operators given by : $\top=1, \perp=0$, $e_{1} \wedge e_{2}=e_{1} e_{2}, e_{1} \vee e_{2}=e_{1}+e_{2}-e_{1} e_{2}$ and $\neg e=1-e$. We write $e_{1} \leq e_{2}$ iff $e_{1} \wedge e_{2}=e_{1}$ and $e_{1} \vee e_{2}=e_{2}$

A sieve $S$ on an object $C$ is a set of morphisms with codomain $C$ such that if $g \in S$ and $\operatorname{cod}(h)=\operatorname{dom}(g)$ then $g h \in S$. A cosieve is defined dually to a sieve. A sieve $S$ is said to cover a morphism $f: D \rightarrow C$ if $f^{*}(S)=\{g \mid \operatorname{cod}(g)=D, f g \in S\}$ contains a cover of $D$. Dually, a cosieve $M$ on $C$ is said to cover a morphism $g: C \rightarrow D$ if the sieve dual to $M$ covers the morphism dual to $g$.

Definition 8.1 (Closed cosieve). A sieve $M$ on an object $C$ of $\mathscr{C}$ is closed if for all $f$ with $\operatorname{cod}(f)=C$ if $M$ covers $f$ then $f \in M$. A closed cosieve on an object $C$ of $\mathscr{C}^{o p}$ is the dual of a closed sieve in $\mathscr{C}$.

Fact 8.2 (Subobject classifier). The subobject classifier in the category of sheaves on a site $(\mathscr{C}, \mathbf{J})$ is the presheaf $\Omega$ where for an object $C$ of $\mathscr{C}$ the set $\Omega(C)$ is the set of closed sieves on $C$ and for each $f: D \rightarrow C$ we have a restriction map $M \mapsto\{h \mid \operatorname{cod}(h)=D, f h \in M\}$.

Lemma 8.3. Let $R$ be an object of $\mathscr{R} \mathscr{A}_{K}$. If $R$ is a field the closed cosieves on $R$ are the maximal cosieve $\{f \mid \operatorname{dom}(f)=R\}$ and the minimal cosieve $\{R \rightarrow 0\}$.

Proof. Let $S$ be a closed cosieve on $R$ and let $\varphi: R \rightarrow A \in S$ and let $I$ be a maximal ideal of $A$. If $A$ is nontrivial we have a field morphism $R \rightarrow A / I$ in $S$ where $A / I$ is a finite field extension of $R$. Let $A / I=R\left[a_{1}, \ldots, a_{n}\right]$. But then the morphism $\vartheta: R \rightarrow R\left[a_{1}, \ldots, a_{n-1}\right]$ is covered by $S$. Thus $\vartheta \in S$ since $S$ is closed. By induction on $n$ we get that a field automorphism $\eta: R \rightarrow R$ is in $S$ but then by composition of $\eta$ with its inverse we get that $1_{R} \in S$. Consequently, any morphism with domain $R$ is in $S$.

Corollary 8.4. For an object $R$ of $\mathscr{R} \mathscr{A}_{K}$. If $R$ is a field, then $\Omega(R)$ is a 2-valued boolean algebra. 
Proof. This is a direct Corollary of Lemma 8.3 . The maximal cosieve $\left(1_{R}\right)$ correspond to the idempotent 1 of $R$, that is the idempotent $e$ such that, $\operatorname{ker} 1_{R}=\langle 1-e\rangle$. Similarly the cosieve $\{R \rightarrow 1\}$ correspond to the idempotent 0 .

Corollary 8.5. For an object $A$ of $\mathscr{R} \mathscr{A}_{K}, \Omega(A)$ is isomorphic to the set of idempotents of $A$ and the Heyting algebra structure of $\Omega(A)$ is the boolean algebra of idempotents of $A$.

Proof. Classically a finite dimension regular algebra over $K$ is isomorphic to a product of field extensions of $K$. Let $A$ be an object of $\mathscr{R} \mathscr{A}_{K}$, then $A \cong F_{1} \times \ldots \times F_{n}$ where $F_{i}$ is a finite field extension of $K$. The set of idempotents of $A$ is $\left\{\left(d_{1}, \ldots, d_{n}\right) \mid 1 \leq j \leq n, d_{j} \in F_{j}, d_{j}=0\right.$ or $\left.d_{j}=1\right\}$. But this is exactly the set $\Omega\left(F_{1}\right) \times \ldots \times \Omega\left(F_{n}\right) \cong \Omega(A)$. It is obvious that since $\Omega(A)$ is isomorphic to a product of boolean algebras, it is a boolean algebra with the operators defined pointwise.

Theorem 8.6. The topos $\operatorname{Sh}\left(\mathscr{R} \mathscr{A}_{K}^{o p}, \mathbf{J}\right)$ is boolean.

Proof. The subobject classifier of $\operatorname{Sh}\left(\mathscr{R} \mathscr{A}_{K}^{o p}, \mathbf{J}\right)$ is $1 \stackrel{\text { true }}{\longrightarrow} \Omega$ where for an object $A$ of $\mathscr{R} \mathscr{A}_{K}$ one has $\operatorname{true}_{A}(*)=1 \in A$.

It is not possible to show that the topos $\operatorname{Sh}\left(\mathscr{R}_{A_{K}^{o p}}^{o}, \mathbf{J}\right)$ is boolean in an intuitionistic metatheory as we shall demonstrate. First we recall the definition of the Limited principle of omniscience (LPO for short). Definition 8.7 (LPO). For any binary sequence $\alpha$ the statement $\forall n[\alpha(n)=0] \vee \exists n[\alpha(n)=1]$ holds.

LPO cannot be shown to hold intuitionistically. One can, nevertheless, show that it is weaker than the law of excluded middle [2].

Theorem 8.8. Intuitionistically, if $\operatorname{Sh}\left(\mathscr{R} \mathscr{A}_{K}^{o p}, \mathbf{J}\right)$ is boolean then LPO holds.

Proof. Let $\alpha \in K[[X]]$ be a binary sequence. By Lemma 5.7 one has an isomorphism $\Lambda: \mathbf{F}[[X]] \stackrel{\sim}{\longrightarrow} \mathbf{F}^{\widetilde{\mathbf{N}}}$.

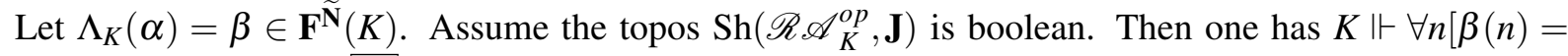
$0] \vee \exists n[\beta(n)=1]$. By $\vee$ this holds only if there exist a cocover of $K$

$$
\left\{\vartheta_{i}: K \rightarrow A_{i} \mid i \in I\right\} \cup\left\{\xi_{j}: K \rightarrow B_{j} \mid j \in J\right\}
$$

such that $B_{j} \Vdash \forall n\left[\left(\beta \xi_{j}\right)(n)=0\right]$ for all $j \in J$ and $A_{i} \Vdash \exists n\left[\left(\beta \vartheta_{i}\right)(n)=1\right]$ for all $i \in I$. Note that at least one of $I$ or $J$ is nonempty since $K$ is not covered by the empty cover.

For each $i \in I$ there exist a cocover $\left\{\eta_{\ell}: A_{i} \rightarrow D_{\ell} \mid \ell \in L\right\}$ of $A_{i}$ such that for all $\ell \in L$, we have $D_{\ell} \Vdash\left(\beta \vartheta_{i} \eta_{\ell}\right)(m)=1$ for some $m \in \widetilde{\mathbf{N}}\left(D_{\ell}\right)$. Let $m=\sum_{t \in T} e_{t} n_{t}$ then we have a cocover $\left\{\xi_{t}: D_{\ell} \rightarrow C_{t}=\right.$ $\left.D_{\ell} /\left\langle 1-e_{t}\right\rangle \mid t \in T\right\}$ such that $C_{t} \Vdash\left(\beta \vartheta_{i} \eta_{\ell} \xi_{t}\right)\left(n_{t}\right)=1$ which implies $\xi_{t} \eta_{\ell} \vartheta_{i}\left(\alpha\left(n_{t}\right)\right)=1$. For each $t$ we can check whether $\alpha\left(n_{t}\right)=1$. If $\alpha\left(n_{t}\right)=1$ then we have witness for $\exists n[\alpha(n)=1]$. Otherwise, we have $\alpha\left(n_{t}\right)=0$ and $\xi_{t} \eta_{\ell} \vartheta_{i}(0)=1$. Thus the map $\xi_{t} \eta_{\ell} \vartheta_{i}: K \rightarrow C_{t}$ from the field $K$ cannot be injective, which leaves us with the conclusion that $C_{t}$ is trivial. If for all $t \in T, C_{t}$ is trivial then $D_{\ell}$ is trivial as well. Similarly, if for every $\ell \in L, D_{\ell}$ is trivial then $A_{i}$ is trivial as well. At this point one either have either (i) a natural number $m$ such that $\alpha(m)=1$ in which case we have a witness for $\exists n[\alpha(n)=0]$. Or (ii) we have shown that for all $i \in I, A_{i}$ is trivial in which case we have a cocover $\left\{\xi_{j}: K \rightarrow B_{j} \mid j \in J\right\}$ such that $B_{j} \Vdash \forall n\left[\left(\beta \xi_{j}\right)(n)=0\right]$ for all $j \in J$. Which by LC means $K \Vdash \forall n[\beta(n)=0]$ which by $\forall$ means that for all arrows $K \rightarrow R$ and elements $d \in \tilde{\mathbf{N}}(R), R \Vdash \beta(d)=0$. In particular for the arrow $K \stackrel{1_{K}}{\longrightarrow} K$ and every natural number $m$ one has $K \Vdash \beta(m)=0$ which implies $K \Vdash \alpha(m)=0$. By $\equiv$ we get that $\forall m \in \mathbb{N}[\alpha(m)=0]$. Thus we have shown that LPO holds.

Corollary 8.9. It cannot be shown in an intuitionistic metatheory that the topos $\operatorname{Sh}\left(\mathscr{R} \mathscr{A}_{K}^{o p}, \mathbf{J}\right)$ is boolean. 


\section{References}

[1] Steven Awodey (1997): Logic in topoi: Functorial Semantics for Higher-Order Logic. Ph.D. thesis, The University of Chicago.

[2] Douglas Bridges \& Fred Richman (1987): Varieties of Constructive Mathematics. Lecture note series, Cambridge University Press, doi $10.1017 /$ cbo9780511565663.

[3] Thierry Coquand, Henri Lombardi \& Claude Quitté (2010): Curves and coherent Prüfer rings. J. Symb. Comput. 45(12), pp. 1378-1390, doi 10.1016/j.jsc.2010.06.016

[4] Michel Coste, Henri Lombardi \& Marie-Françoise Roy (2001): Dynamical method in algebra: effective Nullstellensätze. Annals of Pure and Applied Logic 111(3), pp. 203 - 256, doi:10.1016/S0168-0072(01)00026-4.

[5] Jean Della Dora, Claire Dicrescenzo \& Dominique Duval (1985): About a new method for computing in algebraic number fields. In Bob Caviness, editor: EUROCAL '85, Lecture Notes in Computer Science 204, Springer Berlin / Heidelberg, pp. 289-290, doi 10.1007/3-540-15984-3_279.

[6] A. Fröhlich \& J. C. Shepherdson (1956): Effective Procedures in Field Theory. Philosophical Transactions of the Royal Society of London. Series A, Mathematical and Physical Sciences 248(950), pp. 407-432, doi $10.1098 /$ rsta.1956.0003

[7] Peter T. Johnstone (2002): Sketches of an Elephant: A Topos Theory Compendium - Volume 2. Oxford Logic Guides 44, Oxford University Press.

[8] John F. Kennison (1982): Separable algebraic closure in a topos. Journal of Pure and Applied Algebra 24(1), pp. 7 - 24, doi $10.1016 / 0022-4049(82) 90055-X$

[9] Henri Lombardi \& Claude Quitté (2011): Algèbre Commutative, Méthodes Constructives. Mathématiques en devenir, Calvage et Mounet.

[10] Saunders MacLane \& Ieke Moerdijk (1992): Sheaves in Geometry and Logic: A First Introduction to Topos Theory, corrected edition. Springer, doi 10.1007/978-1-4612-0927-0

[11] Michael Makkai \& Gonzalo E. Reyes (1977): First order categorical logic: model-theoretical methods in the theory of topoi and related categories. Lecture notes in mathematics 611, Springer-Verlag, doi $10.1007 / \mathrm{BFb} 0066201$.

[12] Bassel Mannaa \& Thierry Coquand (2013): Dynamic Newton-Puiseux theorem. J. Logic \& Analysis 5, doi $10.4115 /$ jla.2013.5.5

[13] Per Martin-Löf (1972): An intuitionistic theory of types. Reprinted in Twenty-five years of constructive type theory, Oxford University Press, 1998, 127-172.

[14] Ray Mines, Fred Richman \& Wim Ruitenburg (1988): A course in constructive algebra. Universitext (1979), Springer-Verlag, doi:10.1007/978-1-4419-8640-5.

[15] Andrej Ščedrov (1984): Forcing and classifying topoi. Memoirs of the AMS 48, American Mathematical Society (AMS), doi:10.1090/memo/0295. 